\title{
Loss of electrophoretic variation in serially bottlenecked populations
}

\author{
Steven A. McCommas* and \\ Edwin H. Bryant $\dagger \ddagger$
}

* Department of Biological Sciences, Southern Illinois University, Edwardsville, IL 62026. $\dagger$ Department of Biology, University of Houston, Houston, TX 77204-5513

Experimental bottleneck lines were generated from a single outbred population of the housefly using one, four, or 16 pairs of flies. These lines were followed through one, three, or five successive founder-flush cycles. After each flush 30 individuals from each line were assayed for electrophoretic variation at four initially polymorphic loci. Electrophoretic variation largely followed neutral expectation, the major exception being that the differentiation among 16-pair lines was greater than expected. All bottleneck sizes showed significant losses in genetic variation compared to the control, particularly for losses in numbers of alleles. These findings contrast theoretical predictions in that few lines and loci would be required to detect historical bottlenecks.

\section{INTRODUCTION}

When an outbred population passes through a bottleneck genetic variation should decline in direct proportion to the severity of the bottleneck (Chakraborty and Nei, 1977; Maruyama and Fuerst, 1984, 1985; Nei et al., 1975). These predictions suggest that one can use observed losses in heterozygosity and/or number of alleles to infer historical bottlenecks in natural populations. However, because of the large expected sampling variance among loci ( Nei and Roychoudhury, 1974; Fuerst and Maruyama, 1986) many loci might have to be sampled to detect differences in level of average variation between populations. Moreover, random linkage associations within populations and differential level of inbreeding among populations will further inflate sampling variances for genetic variation (Avery and Hill, 1977; Cockerham and Weir, 1983 et al., 1980), so any given set of loci and/or lines could easily deviate from expected losses by chance alone (Fuerst and Maruyama, 1986; Lynch, 1988; Nei and Roychoudhury, 1974).

Nevertheless studies have used reduced genetic variation at moderately few electrophoretic loci to infer historical bottlenecks in natural populations (e.g., Bryant, 1984; Bryant et al., 1981; Johnson,

$\ddagger$ To whom correspondence should be addressed
1987; O'Brien et al., 1987; Schwaegerle and Schaal, 1979; Taylor and Gorman, 1975). The practice of using reduced genetic variation to infer putative bottlenecks will likely continue, particularly using electrophoretic loci, so it is important to evaluate the extent the reduction in genetic variation due to bottlenecks can be detected in natural populations. It is unlikely that a full theoretical treatment will be forthcoming, given the complexities of selection, hitchhiking, and linkage, so that an empirical check on the reliability of existing models to predict changes in genetic variation is warranted. However, this requires genetic data on populations of precisely known ancestry, and few such data exist.

In a study examining changes in variation of quantitative traits in experimental populations of the housefly, Musca domestica L., that were serially bottlenecked (Bryant et al., 1986a,b) we also examined electrophoretic variation at four loci (that were initially polymorphic in the ancestral line) in these same lines. Evidence from both quantitative trait variation and measures of fitness indicates that selection occurred within these lines during the course of the serial founder-flush cycles (Bryant et al., 1986a, b; Bryant et al., 1989). In addition because the housefly has only five autosomal pairs of chromosomes considerable linkage disequilibria should be generated as a result of a bottleneck (Avery and Hill, 1977; Franklin and 
Lewontin, 1970). Hence, we might predict little correspondence between observed and theoretical changes in variation in these lines. These data thus allow a test of how well limited electrophoretic data may reflect historical bottlenecks in a case where the effects of linkage and selection should be pronounced. We specifically address two questions. First, can electrophoretic variation be used to detect known bottlenecks in experimental populations? Secondly, how well do losses in variation follow neutral expectation?

\section{MATERIALS AND METHODS}

An initial outbred population of the housefly was established in the laboratory by taking a single sample of flies ( $>100$ females) from a refuse site in Alvin, TX. In the third generation in the laboratory, four replicate bottleneck lines were established from this outbred line, for each of three bottleneck sizes, by using offspring from one, four, or 16 pairs of flies. After the populations increased to normal size (about 1000 pairs) samples of thirty adult flies were taken from each line and frozen at $-70^{\circ} \mathrm{C}$ until electrophoresis. This procedure was repeated for a total of three successive founder-flush cycles for each line. Two of these replicate lines per bottleneck size were further subjected to two additional founder-flush episodes, totalling five founder-flush cycles for these lines.

Horizontal electrophoresis was carried out using 12 per cent $(\mathrm{w} / \mathrm{v})$ starch gels (Electrostarch, Lot 392) with apparatus and procedures as described in Selander et al. (1971), Brewer (1970), and Shaw and Prasad (1970). Four polymorphic enzymes were assayed for each individual fly: two phosphoglucomutases (PGM-1, PGM-2; EC 2.7.5.1), an esterase (EST; EC 3.1.1.1), and a superoxide dismutase (SOD; EC 1.15.1.1). Progeny tests were used to confirm Mendelian inheritance of electromorphs. The esterase locus had three alleles, one of which was null, and estimates of allele frequencies used Berstein's correction (Cavalli-Sforza and Bodmer, 1971, p. 55). The first measure of genetic variation was the average heterozygosity computed for each loccus as $H=$ $1-\sum x(i)^{2}$, where $x(i)$ is the frequency of the $i$ th allele, and then these individual $H$ values were averaged over the four loci. Assuming rapid rate of return to equilibrium after a bottleneck the expected loss in heterozygosity would be (1$1 / 2 N$ ) per bottleneck episode (where $N$ is the bottleneck number of individuals), so observed values can be compared directly to this expectation. For multiple bottlenecks an average rate of decay of heterozygosity was obtained as the slope of the linear regression of $\log _{\mathrm{e}}\left[\mathrm{H}_{\mathrm{t}} / \mathrm{H}_{0}\right]$ onto bottleneck episode number with the statistical constraint of the regression to pass through the origin (Bliss, 1967; $H_{0}$ and $H_{\mathrm{t}}$ are the respective heterozygosities observed initially and after the $t$ th founder-flush cycle). The residuals about these linear regressions can be used in significance testing but the errors may not be independent (e.g., Felsenstein, 1985; Hill, 1972a, $b$ ). A more conservative and unbiased test is to convert these regression slopes back into expected losses in heterozygosity per line after the specified number of bottleneck episodes (i.e., three or five). These single values per line can be used in significance tests comparing observed to expected levels of variation (i.e., $n=4$ or $n=2$ lines per bottleneck episode). For the bottleneck lines subjected to five successive bottleneck episodes separate regression estimates were made for the loss rate up to the third bottleneck and then over the five bottlenecks.

A second measure of variation was $n$, the observed number of alleles per locus. The expected rate of loss in numbers of alleles is conditional on the initial number of alleles as well as their frequencies (Maruyama and Fuerst, 1985), so a general formula relating loss in alleles to bottleneck size is not possible. To determine expected loss in numbers of alleles per locus requires use of a multinomial distribution to enumerate all possible outcomes of sampling (Denniston, 1978; Engels, 1980), so one needs to know the frequencies of alleles after each successive bottleneck episode as a basis of determining expected losses for the next episode. In order to generate these expected losses in number of alleles we adopted a bootstrap approach (Efron, 1982; Efron and Gong, 1983) whereby a sample of $2 N$ genes was drawn from an infinite population with the number and frequency of alleles available equal to that in the base population. Once the number and frequency of the alleles in the sampled population were determined, these were used as the basis for the next sampling cycle (bottleneck episode). The process was repeated for 1000 sequences of five successive bottleneck episodes starting with the same initial number of alleles and allele frequencies as in the base population. These were averaged to obtain a single expectation for each bottleneck size over the successive bottleneck episodes that was compared to the observed losses in the same manner as for heterozygosities. 
RESULTS

There were 13 initial alleles over the four loci in the control population, and no allele was lost in the control line over the course of the experiment. As a check for possible changes in the frequencies of alleles in response to the laboratory environment per se independent of bottleneck effects, we analyzed the control population at the start of the experiment and after each bottleneck episode. Levels of heterozygosity per enzyme and bottleneck episode for the control are shown in fig. 1. No monotonic changes are evident over the course of the experiment for any of the loci, and only

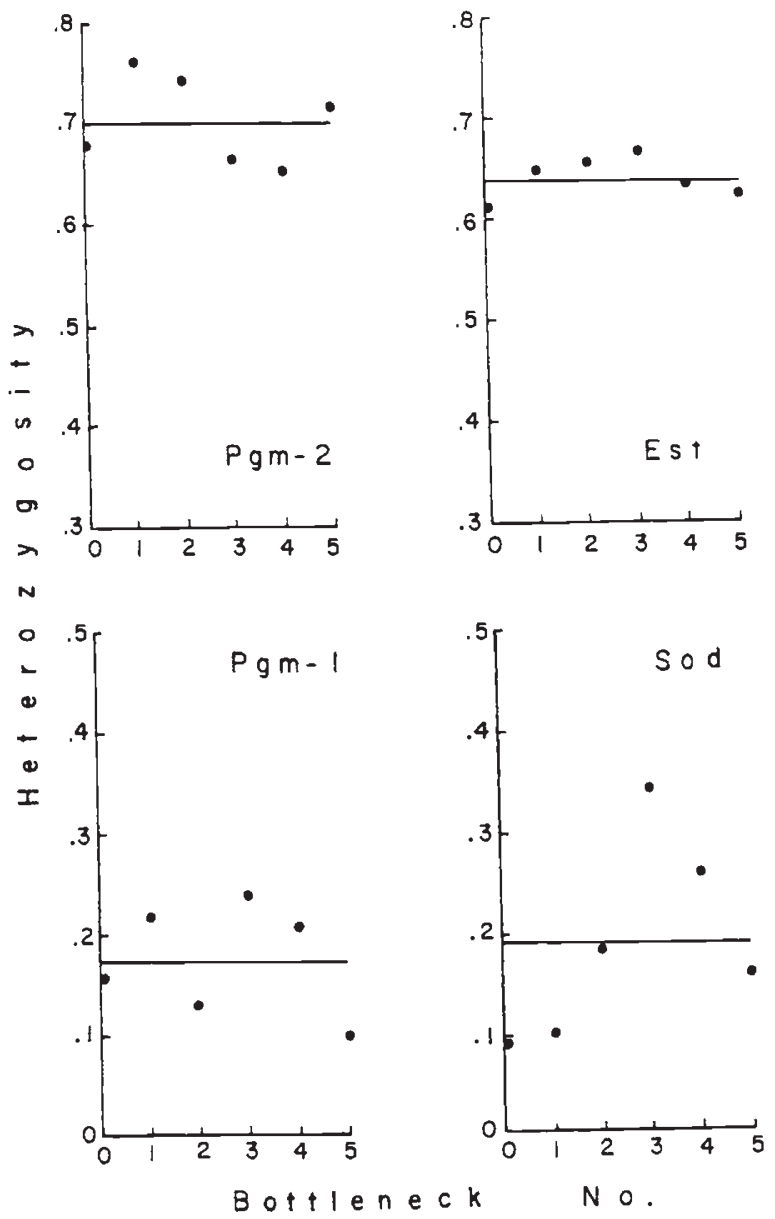

Figure 1 Levels of heterozygosity for the control line at the start of experiment and after each of the five consecutive bottleneck episodes at each polymorphic locus. Horizontal line indicates the average of the six values. Only the variation in heterozygosity for Sod was significantly greater than neutral expectation, based on the expected sampling variance of average heterozygosity of Nei and Roychoudhurdy (1974).
SOD showed significantly greater variance over time $(P<0.025)$ compared with the expected variance in heterozygosity given by Nei and Roychoudhury (1974). Since there was no clear trend in heterozygosity change over time even for this locus, we utilized averages over the six tests (one initial and five bottleneck episodes) as initial heterozygosities at each locus in evaluating bottleneck effects.

The proportional losses in heterozygosity for the bottleneck lines in relation to the control and to neutral expectation are given in table 1 for the three bottleneck sizes after one, three, or five successive bottleneck episodes. Significant losses in heterozygosity were detected for the single-pair lines after the first, third, and fifth bottleneck cycles $\left(t_{\mathrm{a}}\right.$ in table 1$)$. For the larger bottleneck sizes, the only significant losses were observed for the fourpair lines after the first bottleneck. No significant deviations from neutral expectation were detected for any bottleneck size or founder-flush cycle $\left(t_{\mathrm{b}}\right.$ in table 1).

Interestingly, after the fifth bottleneck the level of heterozygosity for the four-pair lines was as high as that for the control, despite being significantly lower in heterozygosity after the first bottleneck (table 1). This was not a consequence of loss of replicate lines: In examining the two replicate lines that were assayed after the fifth bottleneck, there was still significantly lower heterozygosity after the first bottleneck in these lines. Since there was a significant loss in numbers of alleles in these lines (see below), there was an apparent reshuffling of frequencies of remaining alleles in these lines that recovered heterozygosity to the level of the control.

Proportional losses in numbers of alleles in relation to the control and to neutral expectation are shown in table 2 , for the three bottleneck sizes. Significant losses in numbers of alleles were obtained for all bottleneck sizes, after one, three, or five bottleneck episodes ( $t_{\mathrm{a}}$ in table 2$)$. For the single-pair or four-pair lines there was no significant departure of observed losses from neutral expectation, whereas for the 16-pair lines there was a significantly greater loss in number of alleles over expectation after both the first and third bottleneck episodes ( $t_{\mathrm{b}}$ in table 2 ).

As genetic variation erodes within bottleneck populations it should be transformed into genetic variation among populations in proportion to bottleneck size (Wright, 1951; Falconer, 1981). Specifically, the variance among bottleneck lines of size $2 N$ would be $2 F S^{2}=S^{2} / N$ where $S^{2}$ is the initial [additive] genetic variation at a particular 
locus within the base population, provided the lines increase in size rapidly after each bottleneck (e.g., Lynch, 1988). When sample sizes are large $\left[n(n-1) S_{\mathrm{B}}^{2}\right] / S^{2}$ would be distributed approximately as chi square with $n-1$ degrees of freedom, where $S_{\mathrm{B}}^{2}$ is the genetic variance among lines of equal bottleneck size (e.g., Lande, 1977). The chi square values for the four loci and three bottleneck sizes are given in table 3 . Only the chi squares for the 16-pair lines were significantly different from expectation for any locus. Hence, genetic differentiation among lines was consistent with neutral expectation for the single-pair and fourpair lines but not for the 16-pair lines, in which significantly greater differentiation among lines occurred than expected by random drift.

To determine if any loci were linked, progeny from 38 single-pair matings were obtained, and

Table 1 Proportion of heterozygosity for bottleneck lines in relation to control after one, three, or five bottleneck episodes for the three bottleneck sizes of one, four, or 16 pairs of flies

\begin{tabular}{|c|c|c|c|c|}
\hline \multirow{2}{*}{$\begin{array}{l}\text { Bottleneck } \\
\text { episode }\end{array}$} & & \multicolumn{3}{|c|}{ Bottleneck size (No. of pairs) } \\
\hline & & 1 & 4 & 16 \\
\hline $\begin{array}{l}1 \\
(n=4)\end{array}$ & $\begin{array}{l}\text { Observed proportion } \\
\text { Expected proportion } \\
t_{\mathrm{a}} \\
t_{\mathrm{b}}\end{array}$ & $\begin{array}{l}0.681 \\
0 \cdot 750 \\
4.99^{*} \\
0.64 \mathrm{~ns}\end{array}$ & $\begin{array}{l}0 \cdot 910 \\
0.938 \\
3 \cdot 46^{*} \\
0 \cdot 59 \mathrm{~ns}\end{array}$ & $\begin{array}{l}0.986 \\
0.984 \\
1.53 \mathrm{~ns} \\
0.12 \mathrm{~ns}\end{array}$ \\
\hline $\begin{array}{l}3 \\
(n=4)\end{array}$ & $\begin{array}{l}\text { Observed proportion } \\
\text { Expected proportion } \\
t_{\mathrm{a}} \\
t_{\mathrm{b}}\end{array}$ & $\begin{array}{l}0 \cdot 386 \\
0 \cdot 422 \\
7 \cdot 89^{* *} \\
0 \cdot 32 \text { ns }\end{array}$ & $\begin{array}{l}0.891 \\
0.824 \\
3 \cdot 11 \mathrm{~ns} \\
0.90 \mathrm{~ns}\end{array}$ & $\begin{array}{l}0.934 \\
0.954 \\
3 \cdot 09 \mathrm{~ns} \\
0 \cdot 51 \mathrm{~ns}\end{array}$ \\
\hline $\begin{array}{l}5 \\
(n=2)\end{array}$ & $\begin{array}{l}\text { Observed proportion } \\
\text { Expected proportion } \\
t_{\mathrm{a}} \\
t_{\mathrm{b}}\end{array}$ & $\begin{array}{c}0.284 \\
0 \cdot 237 \\
15 \cdot 21^{*} \\
0 \cdot 81 \mathrm{~ns}\end{array}$ & $\begin{array}{l}1.006 \\
0.724 \\
0.00 \mathrm{~ns} \\
8.33 \mathrm{~ns}\end{array}$ & $\begin{array}{l}0.921 \\
0.924 \\
4.22 \mathrm{~ns} \\
0.02 \mathrm{~ns}\end{array}$ \\
\hline
\end{tabular}

$t_{\mathrm{a}}=$ student $t$ value of observed vs. control; $t_{\mathrm{b}}=$ student $t$ value of observed vs. expected proportion based on neutral expectation. All tests based on arcsin transformed proportions. $\quad *=P<0.05 . \quad * *=p<0.01$. ns $=$ nonsignificant at $P<0.05$.

Table 2 Proportion of alleles for bottleneck lines in relation to control after one, three, or five bottleneck episodes for the three bottleneck sizes of one, four, or 16 pairs of flies

\begin{tabular}{|c|c|c|c|c|}
\hline \multirow{2}{*}{$\begin{array}{l}\text { Bottleneck } \\
\text { episode }\end{array}$} & & \multicolumn{3}{|c|}{ Bottleneck size (No. of pairs) } \\
\hline & & 1 & 4 & 16 \\
\hline $\begin{array}{l}1 \\
(n=4)\end{array}$ & $\begin{array}{l}\text { Observed proportion } \\
\text { Expected proportion } \\
t_{\mathrm{a}} \\
t_{\mathrm{b}}\end{array}$ & $\begin{array}{c}0.595 \\
0 \cdot 592 \\
11 \cdot 74^{* *} \\
0 \cdot 30 \mathrm{~ns}\end{array}$ & $\begin{array}{l}0 \cdot 846 \\
0 \cdot 854 \\
8 \cdot 81^{* *} \\
0 \cdot 11 \mathrm{~ns}\end{array}$ & $\begin{array}{l}0 \cdot 885 \\
0 \cdot 984 \\
9 \cdot 71^{* *} \\
6 \cdot 18^{* *}\end{array}$ \\
\hline $\begin{array}{l}3 \\
(n=4)\end{array}$ & $\begin{array}{l}\text { Observed proportion } \\
\text { Expected proportion } \\
t_{\mathrm{a}} \\
t_{\mathrm{b}}\end{array}$ & $\begin{array}{c}0 \cdot 438 \\
0 \cdot 390 \\
37 \cdot 81^{* *} \\
2 \cdot 19 \mathrm{~ns}\end{array}$ & $\begin{array}{c}0.731 \\
0.704 \\
10.45^{* *} \\
0.58 \mathrm{~ns}\end{array}$ & $\begin{array}{l}0 \cdot 804 \\
0 \cdot 951 \\
7 \cdot 62^{* *} \\
3 \cdot 98^{*}\end{array}$ \\
\hline $\begin{array}{l}5 \\
(n=2)\end{array}$ & $\begin{array}{l}\text { Observed proportion } \\
\text { Expected proportion } \\
t_{\mathrm{a}} \\
t_{\mathrm{b}}\end{array}$ & $\begin{array}{c}0 \cdot 391 \\
0 \cdot 308 \\
43 \cdot 71^{*} \\
4 \cdot 27 \mathrm{~ns}\end{array}$ & $\begin{array}{c}0 \cdot 756 \\
0 \cdot 616 \\
25 \cdot 24^{*} \\
7 \cdot 40 \mathrm{~ns}\end{array}$ & $\begin{array}{c}0.801 \\
0.896 \\
22 \cdot 41^{*} \\
6 \cdot 41 \mathrm{~ns}\end{array}$ \\
\hline
\end{tabular}

$t_{\mathrm{a}}=$ student $t$ value of observed vs. control; $t_{\mathrm{b}}=$ student $t$ value of observed vs. expected proportion based on neutral expectation. All tests based on arcsin transformed proportions. $*=P<0.05 . \quad * * * P<0.01 .{ }^{* * *}=P<0.001$. ns $=$ nonsignificant at $P>0.05$. 
Table 3 Chi square values for significance of divergence among replicated lines within a bottleneck size after the first bottleneck, in relation to genetic variance within the base populations

\begin{tabular}{llccc}
\hline \multirow{2}{*}{$\begin{array}{l}\text { Bottleneck } \\
\text { size }\end{array}$} & \multicolumn{2}{l}{ Electrophoretic locus } & \\
\cline { 2 - 4 } & $P g m-1$ & $P g m-2$ & Est & Sod \\
\hline 1 & 0.87 & 6.66 & 4.80 & 2.04 \\
4 & 1.86 & 6.06 & 3.57 & 2.07 \\
16 & 1.89 & $13.89^{* * *}$ & $29.55^{* * *}$ & $19.77^{* * *}$ \\
\hline
\end{tabular}

Chi-square tests with $3 \mathrm{df}$.

$* * *=P<0.005$; all other values non-significant at $P>0.05$.

the electrophoretic genotypes were compared between parents and offspring. Departures from expectation assuming independence were tested by chi square, and the only linkage indicated was between $P g m-1$ and $\operatorname{Sod}(P<0 \cdot 004)$. Based upon these small probabilities of independence the two loci appeared to be tightly linked and changes in levels of variation for these two loci would not be independent. In the bottleneck lines there was an apparent linkage between a common allele for $P g m-1$ and a rare allele for Sod and they both increased in frequency during the course of the experiment. As a result the losses in variation at the Pgm-1 locus were mirrored by increases in variation at the Sod locus.

\section{DISCUSSION}

Because the alleles most at risk during a bottleneck episode are those which are initially in low frequency and contribute little to average heterozygosity, the major consequence of a bottleneck is loss in alleles and not in heterozygosity (e.g., Malécot, 1964; Lewontin, 1965). The prediction then is that the losses in numbers of alleles would be the most sensitive indicator of a bottleneck, and our data support this. In the comparisons of losses of variation against the control in tables 1 and 2, there were more cases of significant deviations as well as greater levels of significance attained for losses in numbers of alleles than in losses in heterozygosity.

One possible bias in comparisons of observed losses with those expected under the neutral theory was that the expected losses were assumed to be a direct result of the bottleneck with no additional losses in the period of recovery immediately following the bottleneck. This assumption would result in underestimates of the expected losses; however, the only significant deviations from expectation occurred in the 16-pair lines (table 2). Since the rebound for the 16-pair lines after each bottleneck was extremely rapid and considerably faster than for the single-pair or four-pair lines, these additional losses are unlikely the result of slow population growth after each bottleneck.

For these same lines after the first bottleneck episode we previously found an increase in additive genetic variation within and among lines for eight morphometric traits (Bryant et al., 1986a,b). Since many of these traits were closely associated with fitness by virtue of their correlation to general body size and fecundity they might be expected to show nonadditive components of genetic variation, particularly directional dominance (Bryant et al., $1986 a$ ). The increases in additive genetic variance we observed as a result of the bottleneck treatment were consistent with possible nonadditive components of genetic variance affecting our traits (Goodnight, 1987, 1988). Moreover, over the course of the five bottleneck episodes the inbreeding depression observed after the initial bottleneck (e.g., fig. 3, Bryant et al., 1986) was largely ameliorated in that only one single-pair line among all the lines remained low in fitness by the fifth bottleneck episode (Bryant et al., 1989). In contrast, loss of electrophoretic variation over the course of the experiment seemed to follow neutral expectation for both loss of genetic variance within lines and increase in genetic variance among lines, the major exception being the 16-pair lines.

One is compelled to ask why such differences in responses to bottlenecks could occur among suites of traits within lines. Our populations were allowed to flush after each bottleneck and before sampling for electrophoresis, so that random linkage effects generated by the bottlenecks would be greatly dissipated by the time we sampled our lines (e.g., Lynch, 1988). The consistency of our results to neutral expectation seems to confirm that sufficient independence of these loci from loci affected by other processes, particularly selection, has been achieved. Despite probable selection acting on some parts of the genome (i.e., quantitative traits) electrophoresis was still effective in detecting losses in genetic variation during bottlenecks as predicted by neutral theory.

In an experiment similar to ours, Sing et al. (1973) followed genetic variation at seven electrophoretic loci for 21 generations in lines of Drosophila melanogaster kept at small size by starting each generation with five randomly chosen inseminated females. Four of their seven electrophoretic loci deviated from neutral expectation, and as well, we observed that half of our loci 
deviated from neutrality (Pgm-1, Sod). Despite these deviations, however, the decline in average heterozygosity over all loci in both cases closely followed neutral expectation. Hence, measures of average genetic variation may still be useful in detecting the effects of small population size, even though individual loci may deviate from expectation.

Our significance tests for loss in average genetic variability of bottleneck lines from the ancestral control were based on the observed variance in these losses among our replicate bottleneck lines. The most common question one wants to ask is whether or not a single population could have experienced a bottleneck during its establishment from a putative ancestral population. The variance of average heterozygosity is expected to be inversely proportional to the number of loci (for a given number of individuals; Nei and Roychoudhury, 1974). Our observed variance among replicate lines of [arcsin-transformed] proportion heterozygosity based on 30 individuals, $S_{\mathrm{H}}^{2}$, can then be used to estimate the number of polymorphic loci necessary to detect a bottleneck of a given size in a single derived population (e.g., Sokal and Rohlf, 1977). using $4 S_{\mathrm{H}}^{2}$ as the presumed variance among lines based on a single locus, and an expected decline of heterozygosity of $(1-1 / 2 N)$ for a bottleneck size of $N$ individuals, the number of polymorphic loci necessary to be 80 per cent sure of detecting the bottleneck at the 5 per cent level of significance would be seven, 15, and 46 for bottleneck sizes of one, four, and 16-pair, respectively (assuming electrophoretic samples of 30 individuals). Although no canonical estimates can be similarly generated for number of alleles, since they would depend upon particular allele frequencies, the number of loci needed should be less than that for average heterozygosity. These results suggest that few loci polymorphic in the putative ancestor may be needed to detect bottlenecks when they are severe (i.e., less than ten pairs), even when selection and linkage effects may be pronounced. Electrophoresis thus seems capable of detecting small bottlenecks, which are the ones that would most drastically alter genomic structure and therefore be important to detect.

Acknowledgements We are indebted to L. Meffert, R. Burton, J. Jacobson, and an anonymous reviewer for their valuable comments on an earlier draft of this manuscript. The study was supported by grants from the National Science Foundation (BSR-8198128) and The University of Houston Coastal Center.

\section{REFERENCES}

AVERY, P. J. AND HILL, W. G. 1977. Variability in genetic parameters among small populations. Genet. Res., 29, 193-213.

BLISS, C. I. 1967. Statistics in Biology, vol. I. McGraw-Hill, New York.

BREWER, G. J. 1970. An Introduction to isozyme Technique. Academic Press, New York.

BRYANT, E. H. 1984. A comparison of electrophoretic and morphometric variability in the face fly, Musca autumnalis. Evolution, 38, 455-458.

BRYANT, E. H., AND DIJK, H. AND VAN DELDEN, W. 1981. Genetic variability of the face fly, Musca autumnalis De Geer, in relation to a population bottleneck. Evolution, 35, 872-881.

BRYANT, E. H., McCOMMAS, S. A. AND COMBS, L. M. 1986a. The effect of an experimental bottleneck upon quantitative genetic variation in the housefly. Genetics, 114, 1191-1211.

BRYANT, E. H., COMBS, L. M. AND McCOMMAS, S. A. 1986 b. Morphometric differentiation among experimental lines of the housefly in relation to a bottleneck. Genetics, 114, 1213-1223.

BRYANT, E. H., MEFFERT, L. M. AND McCOMMAS, S. A. 1989. Fitness rebound in serially bottlenecked populations of the housefly. Amer. Nat. (submitted).

CAVALLI-SFORZA, L. L. AND BODMER, W. F. 1971. The Genetics of Human Populations. W. H. Freeman, San Francisco.

CHAKRABORTY, R. AND NEI, M. 1977. Bottleneck effects on average heterozygosity and genetic distance with the stepwise mutation model. Evolution, 31, 347-356.

COCKERHAM, C. C. AND WEIR, B. S. 1983. Variance of actual inbreeding. Theoret. Popul. Biol., 23, 85-109.

DENNISTON, C. 1978. Small population size and genetic diversity. Implications for endangered species. In Temple, S. A. (ed.) Endangered Birds: Management Techniques for Preserving Endangered Species, University of Wisconsin Press, Madison, pp. 281-290.

EFRON, B. 1982. The Jackknife, the Bootstrap and Other Resampling Plans. Soc. Indust. Appl. Math, Philadelphia, PA.

EFRON, B. AND GONG, G. 1983. A leisurely look at the bootstrap, the jackknife and cross-validation. Amer Sci., 37, 36-48.

ENGELS, W. R. 1980. Loss of selectively neutral alleles in small populations and regular mating systems. Theoret. Popul. Biol. 17, 345-364.

FALCONER, D. S. 1981. Introduction to Quantitative Genetics. Longman, New York.

FELSENSTEIN, J. 1985. Phylogenies and the comparative method. Amer. Nat., 125, 1-15.

FRANKLIN, I. R. AND R. C. LEWONTIN. 1970. Is the gene the unit of selection? Genetics, 65, 707-734.

FUERST, P. AND MARUYAMA, T. 1986. Considerations on the conservation of alleles and genic heterozygosity in small managed populations. Zoo Biology, 5, 171-179.

GOODNIGHT, C. J. 1987. On the effect of founder events on epistatic genetic variance. Evolution, 41, 80-91.

GOODNIGHT, C. J. 1988. Epistasis and the effect of founder events on the additive genetic variance. Evolution, 42, 441454.

HILL, W. G. 1972a. Estimation of realized heritabilities from selection experiments. I. Divergent selection. Biometrics, $28,747-765$.

HILL, W. G. $1972 b$. Estimation of realized heritabilities from selection experiments. I1. Selection in one direction. Biometrics, $28,767-780$. 
JOHNSON, M. S. 1987. Founder effects and geographic variation in the land snail Theba pisana. Heredity 61, 133-142.

LANDE, R. 1977. Statistical tests for natural'selection on quantitative characters. Evolution, 31, 442-446.

LANDE, R. 1980. Genetic variation and phenotypic evolution during allopatric speciation. Amer. Nat., 116, 463-479.

LEWONTIN, R. C. 1965. Comment in Baker, H. G. and Stebbins, G. L. (eds) The Genetics of Colonizing species, Academic Press, New York, pp. 481-484.

LYNCH, M. 1988. Design and analysis of experiments on random drift and inbreeding depression. Genetics, 120, 791807.

MALECOT, G. 1964. The Mathematics of Heredity. Translated by D. M. Yermanos. Freeman, San Francisco.

MARUYAMA, T. AND FUERST, P. 1984. Population bottlenecks and nonequilibrium models in population genetics. I. Allele numbers when populations evolve from zero variability. Genetics, 108, 745-763.

MARUYAMA, T. AND FUERST, P. 1985. Population bottlenecks and nonequilibrium models in population genetics. II. Numbers of alleles in a small population that was formed by means of a recent bottleneck. Genetics, 111, 675-689.

NE1, M. AND ROYCHOUDHURY, A. K. 1974. Sampling variances of heterozygosity and genetic distance. Genetics, 76, 379390.

NeI, M., MARUYAMA, T. AND R. CHAKRABORTY. 1975. The bottleneck effect and genetic variability in populations. Evolution, 29, 1-10.
O'BRIEN, S. J., WILDT, D. E., BUSH, M., CARO, T. M., FITZGIBBON, C., AGGINDEY, I. AND R. E. LEAKY. 1987. East African cheetahs: Evidence for two population bottlenecks? Proc. Nat. Acad. Sci. (Wash.), 84, 508-511.

SELANDER, R. K., SMITH, M. H., YANG, S. Y., JOHNSON, W. E. AND GENTRY, J. B. 1971. Biochemical polymorphism and systematics in the genus Peromyscus. I. Variation in the old field mouse (Peromyscus polionotus). Studies in Genetics (Univ. Texas Publ. 7103), 6, 49-90.

SHAW, C. R. AND PRASAD, R. 1970. Starch gel electrophoresis of enzymes-a compilation of recipes. Biochem. Gen., 4, 297-320.

SING, C. F., BREWER, G. J. AND THIRTLE, B. 1973. Inherited biochemical variation in Drosophila melanogaster: Noise or signal? I. Single-locus analysis Genetics 75, 381-404.

SOKAL, R. R. AND F. J. ROHLF. 1977. Biometry. W. H. Freeman, San Francisco.

SCHWAEGERLE, K. E. AND SCHAAL, B. A. 1979. Genetic variability and founder effect in the pitcher plant, Sarracenia purpurea L. Evolution, 33, 1210-1218.

TAYLOR, C. E. AND GORMAN, G. C. 1975. Population genetics of a "colonizing" lizard: natural selection for allozyme morphs in Anolis grahami. Heredity, 35, 241-247.

WEIR, B. S., AVERY, P. J. AND HILL, W. G. 1980. Effect of mating structure on variation in inbreeding. Theoret. Popul. Biol., $18,396-429$.

WRiGHT, S. 1951. The genetical structure of populations. Ann. Eugen., 15, 323-354. 